\title{
Spectrum Power Measurements in 2G and 3G Cellular Phone Bands during the 2006 Football World Cup in Germany
}

\author{
Oliver Holland, Member, IEEE, Pascal Cordier, Markus Muck, Member, IEEE, Laurent Mazet, \\ Clemens Klöck, and Tobias Renk
}

\begin{abstract}
This contribution presents spectrum occupancy measurements which have been performed during the 2006 Football World Cup in Germany, in the cities of Kaiserslautern and Dortmund. The measurements investigate the time-variation of powers in $2 \mathrm{G}$ bands $(900 \mathrm{MHz}$ and $1800 \mathrm{MHz})$, a spectrum bandwidth including $3 G$ bands and an ISM band (2000$2600 \mathrm{MHz})$, and a global band $(400-2600 \mathrm{MHz})$, on the day prior to a match, the day of the match, and during the match. The first intention is to study changes in power levels and subsequently relate this to specific events, such as (i) the start of the match, (ii) half-time, and (iii) the end of the match. A next intention is to investigate the autocorrelation structure of changes in power levels, and relate this to the ease with which dynamic spectrum allocation might be performed. The results that ensue are used to inspire a discussion as to how dynamic spectrum allocation and short-range localized solutions such as IEEE 802.11 basestations might be leveraged to bolster capacity at large-scale events.
\end{abstract}

Index Terms-Spectrum Occupancy, Heterogeneous Networks

\section{INTRODUCTION}

$\mathrm{T}$ HE Mobile and wireless communications landscape is expected to evolve towards a heterogeneous scenario involving a multitude of standards, including classical $2 \mathrm{G}$ and 3G cellular systems, EDGE, HSxPA, existing and future generation WiFi (IEEE $802.11 \mathrm{a} / \mathrm{b} / \mathrm{g} / \mathrm{n}$ ), and existing and future generation WiMAX (IEEE $802.16 \mathrm{e} / \mathrm{m}$ ) systems. To realize progression towards the controlled coexistence of such existing and evolving standards, distributed decision making mechanisms and an inherent system architecture are under investigation within the IST-E2R Project [1], as well as the IEEE P1900.4 standardization Working Group [2].

The advantage of short-range communications systems, such as IEEE $802.11 \mathrm{a} / \mathrm{b} / \mathrm{g} / \mathrm{n}$, lies in the fact that they can be easily deployed at a specific location for any period of time (short-term to long-term) in order to meet peak system

Oliver Holland is with King's College London, Strand, London WC2R 2LS, United Kingdom (e-mail: oliver.holland@kcl.ac.uk)

Markus Muck and Laurent Mazet are with Motorola Labs, Parc Les Algorithmes, 91193 Gif-sur-Yvette, France (e-mail: \{Markus.Muck, Laurent.Mazet\}@motorola.com)

Pascal Cordier is with France Telecom R\&D, Paris, France (e-mail: pascal.cordier@orange-ftgroup.com)

Clemens Kloeck and Tobias Renk are with University of Karlsruhe, Germany (e-mail: \{renk, kloeck\}@int.uni-karlsruhe.de) capacity needs in a heterogeneous systems scenario. Spectrum power measurements during large public events can be expected to give indications of when such additional network capacity might be required and at which instants critical peakdemands may occur from the user side. This is one motivation behind the spectrum occupancy measurements reported in this paper, which have been performed during the 2006 Football World Cup in Germany. Measurements have been made at two locations: (i) Dortmund (Germany vs. Poland match, 1314 June 2006), and (ii) Kaiserslautern (Italy vs. USA match, 16-17 June 2006). This work adds to the knowledge-base of spectrum measurements which have been performed in other contexts, e.g. [3], [4], [5].

This paper is structured as follows. Section II gives further detail on the measurement campaign at the 2006 World Cup. Section III presents some measurement results and analysis, as well as an interpretation of the level of activity related to specific events such as the start of the match, half-time, etc. Section IV presents some general discussion of how such results might be used, before the paper concludes in Section $\mathrm{V}$.

\section{Details of the Measurement CAmpaign}

The spectrum power measurements were been performed with the help of the following equipment:

$\Rightarrow$ The main sensor: a spectrum analyzer associated with the "EM Spy" software, developed at France Telecom for spectrum analysis, monitoring, and data collection,

$\Rightarrow$ Two other sensors used to support the data analysis: A system-specific scanner, as well as a trace mobile.

Spectrum power measurements were taken in the proximity of the stadium in both cases, at the same location on the day prior to the match and the day of the match. The exact locations were: (Dortmund: 51 $30^{\prime} 22.28^{\prime \prime} \mathrm{N}, 7^{\circ} 27^{\prime} 36.87^{\prime \prime} \mathrm{E}$; Kaiserslautern: $\left.\quad 49^{\circ} 25^{\prime} 55.44^{\prime \prime} \mathrm{N}, \quad 7^{\circ} 46^{\prime} 50.40^{\prime \prime} \mathrm{E}\right) . \quad$ The measurement equipment was configured as follows: 
1) To cover GSM 900:

- 400 measurement points (every $200 \mathrm{kHz}$ ) in the range $915 \mathrm{MHz}-995 \mathrm{MHz}$, which covers the GSM downlink band $(925 \mathrm{MHz}-$ $960 \mathrm{MHz}$ ),

- 1 "spectrum image" per second.

$\Rightarrow$ 2) To cover DCS 1800:

- 400 measurement points (every $200 \mathrm{kHz}$ ) in the range $1805 \mathrm{MHz}-1885 \mathrm{MHz}$, which covers the DCS 1800 downlink band (1805 $\mathrm{MHz}-1880 \mathrm{MHz}$,

- 1 "spectrum image" per second.

3) To cover UMTS FDD downlink and WiFi:

- 600 measurement points (every $1 \mathrm{MHz}$ ) in the range $2-2.6 \mathrm{GHz}$, which covers the UMTS FDD downlink band $(2110 \mathrm{MHz}-$ $2170 \mathrm{MHz}$ ) and the $2.4 \mathrm{GHz}$ ISM band,

1 "spectrum image" per second.

4) To cover a global band ( $400 \mathrm{MHz}-2600 \mathrm{MHz})$ :

- 1100 measurement points (every $2 \mathrm{MHz}$ ), which covers: (i) PMR bands (450 $\mathrm{MHz}-$ $470 \mathrm{MHz}$ ), (ii) DVB-T bands (470 MHz $860 \mathrm{MHz}$ ), (iii) bands between GSM and DCS 1800 ,

- 1 "spectrum image" every 4 seconds.

Some analytical treatment of the corresponding results is given in the following section.

\section{Spectrum Measurement Results ANd Discussion}

Figures 1 and 2 present the power level measurement results for the Dortmund match on the 13-14 June 2006 in the $900 \mathrm{MHz}$ band, and the Kaiserslautern match on the 16-17 June 2006 in the $1800 \mathrm{MHz}$ band. These particular measurement sets are chosen as they have proven to be perhaps most interesting. The Figures represent a moving average (of order 60) of the average power per spectrum measurement sub-band, i.e. per $200 \mathrm{kHz}$ in these cases. Power-level statistics for the different times with respect to the match, both for the $900 \mathrm{MHz}$ band in Dortmund and the $1800 \mathrm{MHz}$ band in Kaiserslautern, are also presented in Table 1.

Among other observations, the following can be said of these measurement results:

$\Rightarrow$ In both cases, the band power level increases by approximately $2-3 \mathrm{~dB}$ during the match day compared to the day prior to the event.

$\Rightarrow$ The power level at Dortmund decreases gradually before the start of the match. During the first half of the match, the power level reduces significantly, presumably because people are engrossed in the match. This reduction for both Dortmund and Kaiserslautern is by approximately $2-3 \mathrm{~dB}$. In both cases, the band power level increases considerably at half-time (again by approximately 2-3dB). After

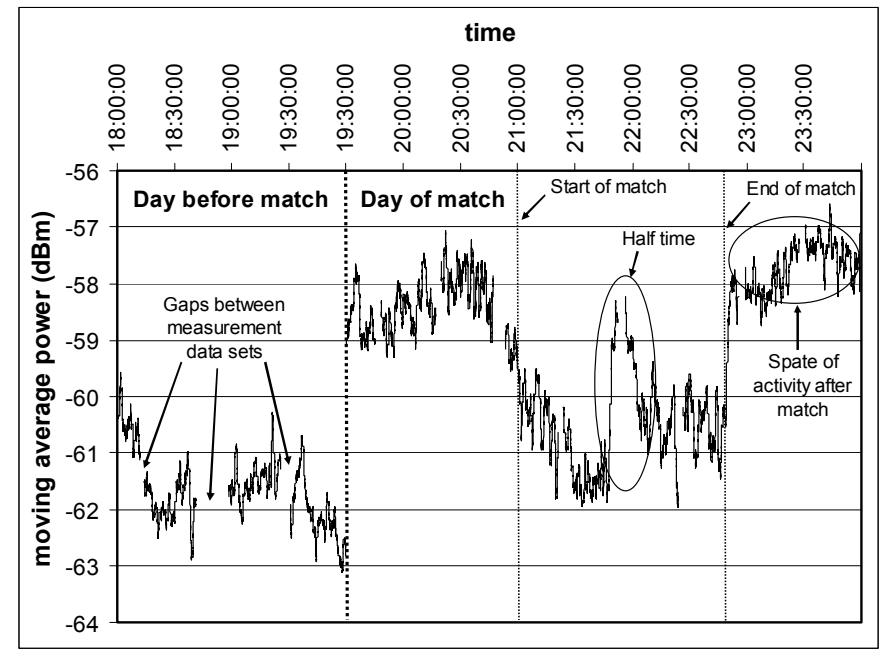

Fig. 1: Time-variation of the average observed power per $200 \mathrm{kHz}$ sub-band in the $900 \mathrm{MHz}$ band in Dortmund

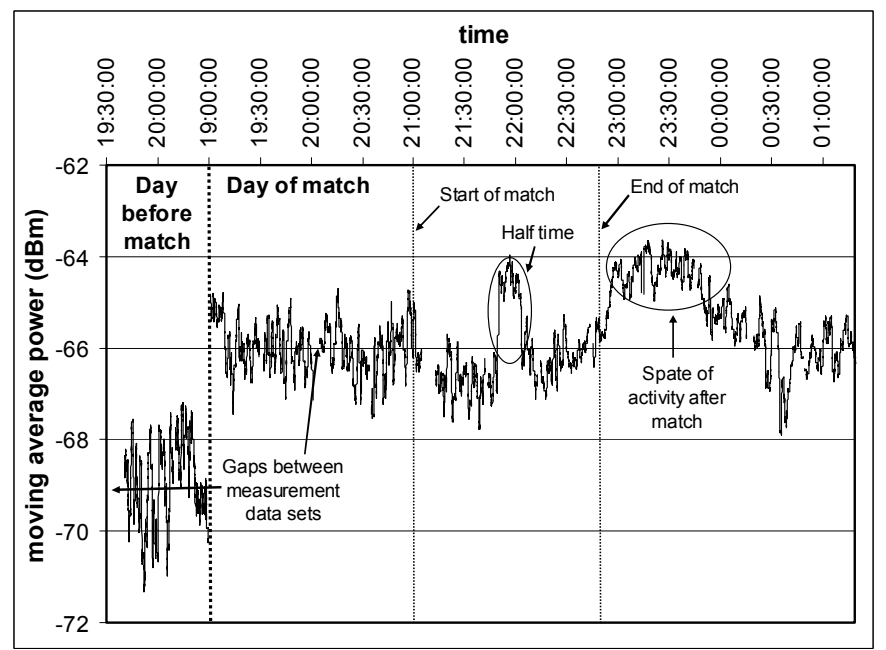

Fig. 2: Time-variation of the average observed power per $200 \mathrm{kHz}$ sub-band in the $1800 \mathrm{MHz}$ band in Kaiserslautern

decreasing again in the second half to roughly the same level as in the first half, the level sharply increases at the end of the match.

$\Rightarrow$ The power level remains very high for at least $60 \mathrm{~min}$ after the match. Note that this level is higher than at any other observed time on the match day. This might be attributed to: (i) people making calls to discuss the match, and (ii) people leaving the ground and making calls as they move to their next destination. Note that the former might also explain the activity at half time.

Notably, trends observed here with respect to the match timing can be easily used by systems to dynamically allocate resources based on predicted load variations. Moreover, statistics such as the power coefficient of variation, also denoted in Table 1, give a rough indication of the confidence with which a dynamic allocation can be made. 
TABLE I

Time-DePendent Statistics ON THE POWER LEVEL PER 200KHz SUB-BAND FOR THE DORTMUND 900MHZ AND KAISERSLAUTERN 1800MHZ BANDS

\begin{tabular}{|c|c|c|c|c|c|c|}
\cline { 2 - 7 } \multicolumn{1}{c|}{} & \multicolumn{2}{c|}{$\begin{array}{c}\text { Average Power } \\
\text { (mW) }\end{array}$} & \multicolumn{2}{c|}{$\begin{array}{c}\text { Average Power } \\
\text { (dBm) }\end{array}$} & \multicolumn{2}{c|}{$\begin{array}{c}\text { Power Coefficient of } \\
\text { Variation }\end{array}$} \\
\cline { 2 - 7 } & $\begin{array}{c}\mathbf{9 0 0 M H z} \\
\text { Dort. }\end{array}$ & $\begin{array}{c}\mathbf{1 8 0 0 M H z} \\
\text { Kaisers. }\end{array}$ & $\begin{array}{c}\mathbf{9 0 0 M H z} \\
\text { Dort. }\end{array}$ & $\begin{array}{c}\mathbf{1 8 0 0 M H z} \\
\text { Kaisers. }\end{array}$ & $\begin{array}{c}\mathbf{9 0 0 M H z} \\
\text { Dort. }\end{array}$ & $\begin{array}{c}\mathbf{1 8 0 0 M H z} \\
\text { Kaisers. }\end{array}$ \\
\hline $\begin{array}{c}\text { Day } \\
\text { Before } \\
\text { Match }\end{array}$ & $6.85 \cdot 10^{-7}$ & $1.31 \cdot 10^{-7}$ & -61.6 & -68.8 & 0.258 & 0.493 \\
\hline $\begin{array}{c}\text { Build- } \\
\text { up to } \\
\text { Match }\end{array}$ & $1.46 \cdot 10^{-6}$ & $2.54 \cdot 10^{-7}$ & -58.4 & -65.9 & 0.339 & 0.276 \\
\hline $\begin{array}{c}\mathbf{1}^{\text {st }} \\
\text { Half }\end{array}$ & $8.39 \cdot 10^{-7}$ & $2.14 \cdot 10^{-7}$ & -60.8 & -66.7 & 0.292 & 0.248 \\
\hline $\begin{array}{c}\text { Half } \\
\text { Time }\end{array}$ & $1.19 \cdot 10^{-6}$ & $3.22 \cdot 10^{-7}$ & -59.2 & -64.9 & 0.299 & 0.260 \\
\hline $\begin{array}{c}\mathbf{2}^{\text {nd }} \\
\text { Half }\end{array}$ & $9.04 \cdot 10^{-7}$ & $2.43 \cdot 10^{-7}$ & -60.4 & -66.1 & 0.283 & 0.225 \\
\hline $\begin{array}{c}\text { Hour } \\
\text { After } \\
\text { Match }\end{array}$ & $1.68 \cdot 10^{-6}$ & $3.64 \cdot 10^{-7}$ & -57.7 & -64.4 & 0.246 & 0.182 \\
\hline
\end{tabular}

It seems logical that proceedings within the match itself should also affect the behavior of users. A good example of this is in the game between Germany and Poland in Dortmund, which Germany won by one goal to nil, where this goal was scored in the 91st minute just before the end of the match. This might partially explain the extremely high power levels after the match. In Kaiserlautern, the sending off of Pablo Mastroeni for the USA just before half time might also have been a factor in the strong increase in power levels at half time.

Next we consider the time-dependence of power levels through studying autocorrelation structures as an indication of the rate of power variability: note that a heavier tail in an autocorrelation vs. time lags plot indicates a tendency for power levels to vary slowly hence more time for the system to react in order to dynamically allocate resources. Using results in Figure 3, we point out some noticeable trends. On the day before the match, the autocorrelation function reduces slowly, only to as much as $\sim 0.35$ within 30 lags ( 30 seconds). During the build-up to the match, the autocorrelation function drops extremely quickly, falling to $\sim 0.1$ within 30 lags, thus indicating that the system must be rapidly reactive if it is going to be able to reallocate resources dynamically based on loads within this period. During the match itself, the autocorrelation function generally drops quite quickly; however, at half time it falls extremely slowly, only to as much as $\sim 0.4$ within 30 lags. This represents a golden opportunity for the benefits of dynamic allocation to be leveraged, especially given the higher power levels observed at half time. Finally, in the hour after the match, the autocorrelation function again drops slowly, also representing a good opportunity for dynamic allocation.

It should be noted that there is a reasonable agreement between the decays of autocorrelation functions for Dortmund and Kaiserslautern. This is perhaps with the exception of during the halves of the actual game.

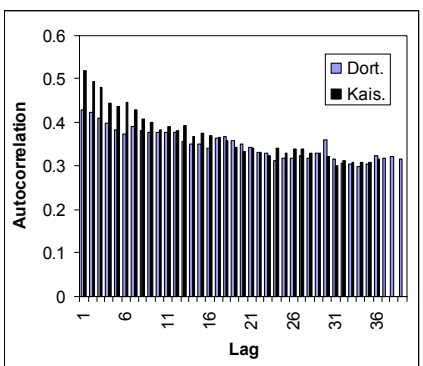

(a)

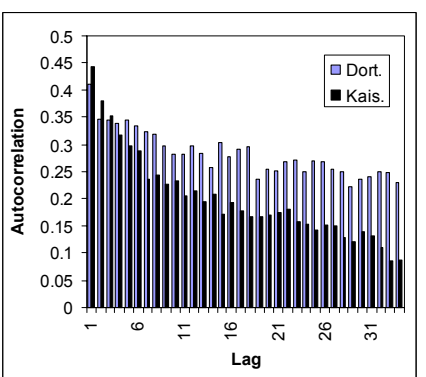

(c)

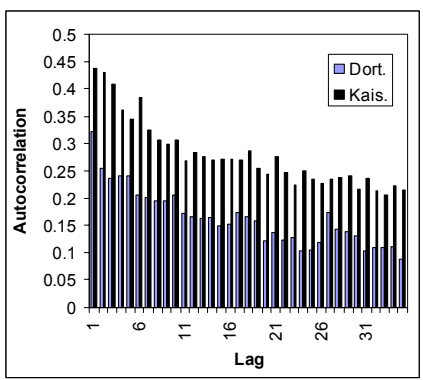

(e)

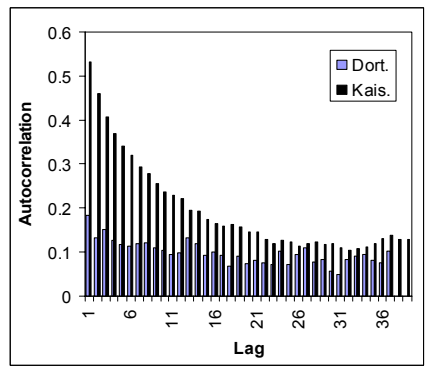

(b)

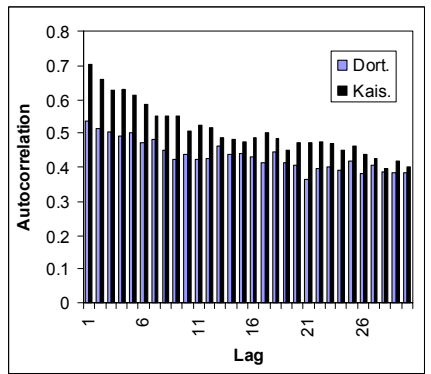

(d)

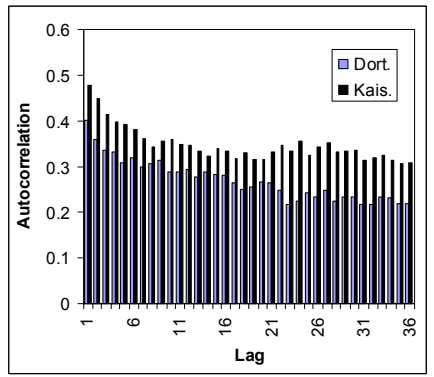

(f)
Fig. 3: Autocorrelation vs. time lags plots for Dortmund and Kaiserslautern: (a) The day before the match, (b) in the build-up to the match, (c) in the first half, (d) at half time, (e) in the second half, and (f) in the hour after the match

\section{Exploitation of the Measurement Results in a HETEROGENEOUS RADIO ENVIRONMENT}

The previous sections have presented a series of measurements giving indications of the usage levels of cellular phone bands during large-scale public events comprising two 2006 Football World Cup matches. The question to be addressed in this section is: how can we derive recommendations for future wireless radio standards from this? It is clear that regulation will undergo enormous changes in the coming years leading to a more flexible spectrum access. Bearing the results of this measurement campaign in mind, it therefore seems quite rational that dynamic access techniques for heterogeneous networks will be a key-issue in future wireless communications.

In the design of future wireless communication systems, it is important to note that the observations in the previous section indicate a high level of activity during predictable events, such just before the start of the match, half-time, and the end of the match. If the capacity of a standard network deployment is insufficient to meet these inherent peak requirements, it would ideally be possible to locally deploy 
additional equipment (eventually run by independent service providers), with this equipment adapting its resource usage strategies according to the predictable events. Such an adaptation might originate in a centralized decision-making approach, with the network controller performing a global resource usage optimization; alternatively, the optimization process might be split among the network and the user entities by application of a distributed decision-making approach, subject to network policies. The latter here is currently under investigation within the IEEE P1900.4 standardization Working Group [2]. Moreover, independently of the optimization approach, the additional equipment may operate in licensed bands (e.g., WiMAX), in unlicensed bands (WiFi, WiMAX, etc.) or in multiple bands simultaneously.

A key requirement with respect to future wireless communications user equipment lies in the (preferably simultaneous) support of multiple heterogeneous Radio Access Technologies (RATs). In order to maximize the number of supported RATs, the user devices preferably must have inherent reconfiguration capabilities, as are currently under investigation within the IST-E ${ }^{2} \mathrm{R}$ II research project [1]. Under these assumptions, a communication initiated by a user device may trigger the following underlying (ideally transparent) operations:

$\Rightarrow$ The user switches his device on and recovers context information (i.e., information on the availability of RATs, expected QoS levels, communication costs, etc.) by dedicated physical pilot channel or a logical channel conveyed by any of the available RATs. In parallel, the user device receives radio resource selection constraints (or policies) subject to which the device will perform the distributed resource selection optimization.

$\Rightarrow$ The user device independently derives its radio resource selection strategy subject to the policies imposed by the network. This selection process may be tightly constrained by the network provider (as is currently the case in the context of cellular radio systems); alternatively, the network may grant increased decision making policies to the terminal in order to (i) assure a higher reactivity of the user devices' resource usage strategies to the time-variant context, (ii) decrease the communication overhead for communicating reconfiguration parameters from the network to user devices, and (iii) reduce the numerical optimization complexity requirements in the network by off-loading parts of this operation to user devices.

$\Rightarrow$ During the periods of average band occupancy, the user devices are expected to maintain links to the $2 \mathrm{G} / 3 \mathrm{G}$ cellular phone access points assuring a high level of QoS. During the peak traffic instants, the cellular network is expected to saturate and the user device will exploit additional resources of lower QoS, such as WiFi, etc. - which typically does not guarantee the final data rate and communication latency. It is also possible that a user device will maintain multiple simultaneous links in order to assure an increased level of availability and higher throughput (4G transition).

\section{CONCLUSION}

The whole landscape of wireless communications will change greatly in the coming years. The considerable demand for bandwidth will increase even further, as will the availability of techniques to meet users' expectations of a seamless access, wherever and whenever they want.

The 2006 Football World Cup in Germany has offered a great opportunity to attain knowledge of the contours of the spectral landscape. Harnessing this opportunity, spectrum measurements in two different locations during the World Cup have been performed, namely Dortmund and Kaiserslautern. It has been seen that power levels in the different cellular systems are generally approximately $2-3 \mathrm{~dB}$ higher on the day of the match. Furthermore, a strong correlation between the process of the match (e.g. build-up, $1^{\text {st }}$ half, half time, etc.) and power levels, can be seen. Basic autocorrelation structure analysis has shown, through differences in the variability of power levels, that greater opportunity for dynamic spectrum allocation exists at certain times within the match.

These measurements form an important background for possible developments in future radio design. Through these measurements it becomes clear that there is great potential for solving occupation bottlenecks through dynamic spectrum access and spectrum sharing techniques. Discussion has also therefore been provided on how such results might be exploited in a heterogeneous radio environment.

\section{ACKNOWLEDGMENTS}

This work has been performed within the project E2RII, which has received funding from the European Community's Sixth Framework programme. This paper reflects only the authors' views; the Community is not liable for any use that may be made of the information contained herein. The contributions of colleagues from the E2RII consortium are hereby acknowledged.

Special thanks must be given to the France Telecom experts that performed the measurements in the field, namely Denis Lafolie, Jean-Jacques Bourhis, Didier Etourneaud, Sylvie Daniaud, and Jean-Luc Garcia. Without their help, it would have been impossible to compile this paper.

\section{REFERENCES}

[1] IST-2005-027714 E² R II, End-to-End Reconfigurability Phase 2 (E ${ }^{2} \mathrm{R}$ II) Project, http://www.e2r2.motlabs.com

[2] IEEE P1900.4 Working Group, http://www.ieeep1900.org

[3] M. McHenry and D. McCloskey, "New York City Spectrum Occupancy Measurements September 2004," Shared Spectrum Company, December 2004

[4] C. L. Holloway et al., "Propagation and Detection of Radio Signals Before, During and After the Implosion of a 13-Story Apartment Building," NIST technical note 1540, May 2005

[5] M. Biggs, A. Henley, and T. Clarkson, "Occupancy Analysis of the 2.4 GHz ISM Band," IEE Proceedings on Communication, Vol. 151, No. 5, October 2004 